\title{
Transcriptome and metabolome reveal redirection of flavonoids in a white testa peanut mutant
}

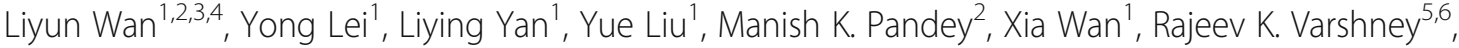 \\ Jiahai Fang ${ }^{2,3,4}$ and Boshou Liao ${ }^{1 *}$
}

\begin{abstract}
Background: Coat color determines both appearance and nutrient quality of peanut. White seed coat in peanut can enhance the processing efficiency and quality of peanut oil. An integrative analysis of transcriptomes, metabolomes and histocytology was performed on wsc mutant and its wild type to investigate the regulatory mechanisms underlying color pigmentation.

Result: Metabolomes revealed flavonoids were redirected in wsc, while multi-omics analyses of wsc mutant seeds and testae uncovered WSC influenced the flavonoids biosynthesis in testa as well as suberin formation, glycolysis, the TCA cycle and amino acid metabolism. The mutation also enhanced plant hormones synthesis and signaling. Further, co-expression analysis showed that FLS genes co-expressed with MBW complex member genes. Combining tissue expression patterns, genetic analyses, and the annotation of common DEGs for these three stages revealed that three testa specific expressed candidate genes, Araip.M7RY3, Aradu.R8PMF and Araip.MHR6K were likely responsible for the white testa phenotype. WSC might be regulated expression competition between FLS and DFR by controlling hormone synthesis and signaling as well as the MBW complex.
\end{abstract}

Conclusions: The results of this study therefore provide both candidate genes and novel approaches that can be applied to improve peanut with desirable seed coat color and flavonoid quality.

Keywords: Peanut (Arachis hypogaea L.), Seed coat, Hormone, Flavonoid, Transcriptional regulation, Metabolome

\section{Background}

Flavonoids are plant polyphenolic secondary metabolites that share a differentially modified but common three ring chemical structure (C6-C3-C6). Thus, on the basis of R1 and R2 site differentiation substitutions within this C6-C3-C6 ring, flavonoids can be classified into at least ten chemical groups, including flavanones, flavones, isoflavonoids, flavans (flavanols), anthocyanins, and flavonols [1]. Anthocyanins are the most conspicuous class of

\footnotetext{
* Correspondence: liaoboshou@163.com

${ }^{1}$ Key Laboratory of Biology and Genetic Improvement of Oil Crops, Ministry of Agriculture, Oil Crops Research Institute of Chinese Academy of Agricultural Sciences, Wuhan, China

Full list of author information is available at the end of the article
}

flavonoids and are responsible for pigmentation in flowers, fruits, seeds, and leaves [2]. Flavonols are the most abundant flavonoids in foods; quercetin, kaempferol, and myricetin are three common isoforms [1]. Flavone is another major class of flavonoids which is commonly conjugated with a sugar group via an Oglycosidic link while some flavones also exist in a Cglycosylated form. In contrast, C-glycosylflavones (also called flavone $\mathrm{C}$-glycosides) comprise an important subgroup of flavonoids that are present in numerous plants [3]; these compounds perform functions in UV protection, caterpillar growth inhibition, pathogen defense, and co-pigments for flower coloration $[4,5]$. 
The flavonoid biosynthetic pathway has been clearly elucidated in model plants; it is well-known that the basic C6-C3-C6 skeleton of these compounds begins with one 4-coumaroyl-CoA and three molecules of malonyl-CoA, catalyzed sequentially by $\mathrm{CHS}, \mathrm{CHI}, \mathrm{F} 3 \mathrm{H}$ and $\mathrm{F}^{\prime} \mathrm{H}$ or $\mathrm{F}^{\prime}{ }^{\prime}{ }^{\prime} \mathrm{H}$ to produce dihydroflavonols (dihydroquercetin, dihydromyricetin and dihydrokaempferol). The dihydroflavonols are then converted to anthocyanidins which are colored but unstable pigments by two reactions catalyzed by DFR and LDOX. DFR catalyzes dihydroquercetin, dihydrokaempferol, and dihydromyricetin to leucocyanidin, leucopelargonidin, and leucodelphinidin, respectively, while LDOX converts the oxidation of leucocyanidin, leucopelargonidin, and leucodelphinidin to cyanidin, pelargonidin, and delphinidin, respectively. Finally, the synthesis of colored and stable anthocyanins confers the glycosylation of cyanidin, pelargonidin, and delphinidin via UFGTs. For PAs, a single cyanidin-3-glucoside and delphinidin-3-glucoside are methylated by MTs to produce peonidin-3-glucoside and petunidin- or malvidin-3-glucoside, respectively. LAR and ANR catalyze leucocyanidin and cyanidin to catechin and epicatechin, respectively. Subsequently, POD and PPOD possibly catalyzed the formation of PA polymers via the catechin or epicatechin with leucocyanidin molecules in vacuolar compartments.

Flavonols are another key flavonoid subgroup which plays pivotal roles in testa pigmentation [6]. Flavonol derivatives have been shown to influence anthocyaninmediated coloration via co-pigmentation effects while FLS catalyzes the conversion of dihydrokaempferol and dihydroquercetin to co-pigment flavonols and thus may influence anthocyanin accumulation levels $[2,7]$.

A number of flavonoid EBGs including CHS, CHI, $F 3 H$, and FLS are transcriptionally regulated by the three R2R3-MYB transcription factors MYB11, MYB12, and MYB111 [8, 9]. Known flavonoid LBGs such as DFR, LDOX, ANR, and TT12 are activated by the MBW ternary transcriptional complex [10]. The crucial regulatory roles played by plant hormones in controlling flavonoid metabolic processes have been elucidated in recent years. The plant hormone JA, for example, is known to induce anthocyanin accumulation in a range of species [11-17], while COI1 regulates the expression of transcription factors, including PAP1, PAP2, and GL3 which themselves mediate the 'late' anthocyanin biosynthetic genes DFR, LDOX, and UF3GT that modulate JAinduced anthocyanin biosynthesis in Arabidopsis [16]. In addition, BR is known to influence JA-induced anthocyanin accumulation by regulating 'late' anthocyanin biosynthesis genes; it is thought that this regulation might be mediated by MBW transcriptional complexes [17].

Anthocyanins, proanthocyanidins, and flavonols are all abundant flavonoids found in the seed coats of numerous plant species $[18,19]$. These compounds not only influence appearance and nutritional qualities but also perform protective roles in the face of microbial pathogens, insect attacks, and against UV light [19, 20]. Generally, almost all natural flavonoids exist in either Oglycoside or C-glycoside forms in plants. Although, dietary flavonoids $\mathrm{O}$-glycoside attract more attention than $\mathrm{C}$-glycoside, C-glycosylflavonoids in most cases tend to have higher antioxidant and anti-diabetes potential than the corresponding O-glycosylflavonoids. The aim of this study is to present the characterization of a white seed coat peanut mutant. Analyses of testae and the seed metabolomes revealed a reprogramming of flavonoid content in the wsc mutant; contents of isoflavones, flavanones, flavones, flavone C-glycosides, and flavonols increased while anthocyanin and PA volumes decreased. The use of transcriptomic profiling revealed downregulation of a subset of genes involved in flavonoid biosynthesis in developing testae in the wsc while key genes involved in flavonol synthesis were enhanced and sucrose and glutamate amino acid metabolism, glycolysis, the TCA cycle, hormone synthesis and signaling were all reprogrammed. The results of this study provide key additional information regarding the mechanisms regulating peanut pigmentation as well as genes that can be utilized for the breeding of white colored testa.

\section{Results}

Phenotypic variation and flavonoid metabolic features

A white seed coat peanut mutant wsc was identified from a mutation population derived from an elite pink seed coat variety Zhonghua 16 (cultivated by Oil Crops Reasearch Institute of Chinese Academy of Agricultural Sciences) once treated with ${ }^{60} \mathrm{Co}$. The WT accumulated pink anthocyanins and no obvious color change was observed in wsc during development (Fig. 1a). A histochemical analysis was therefore performed to determine the nature of polyphenol compounds in developing testae between wsc and WT. TBO staining of developing testae transverse sections revealed the distribution of polymeric phenolic compounds and showed no significant differences between wsc and WT in the three seed development stages assessed (Fig. 1b). A series of flavonoid metabolome profiles were therefore conducted between wsc and WT. We detected a total of 199 flavonoids in testae samples including 19 anthocyanins, 23 flavanones, 63 flavones, 32 flavone C-glycosides, 38 flavonols, three flavonolignans, one hydroxycinnamoyl derivative, 15 isoflavones, and five proanthocyanidins (Additional file 1). Data revealed that the total flavonoid content in wsc was 1.67 times higher than in WT at DAF20, declining to just 1.43 times and 0.97 times that much at DAF40 and DAF60, respectively (Fig. 1c). It is clear that both wsc and WT accumulated flavonoids 


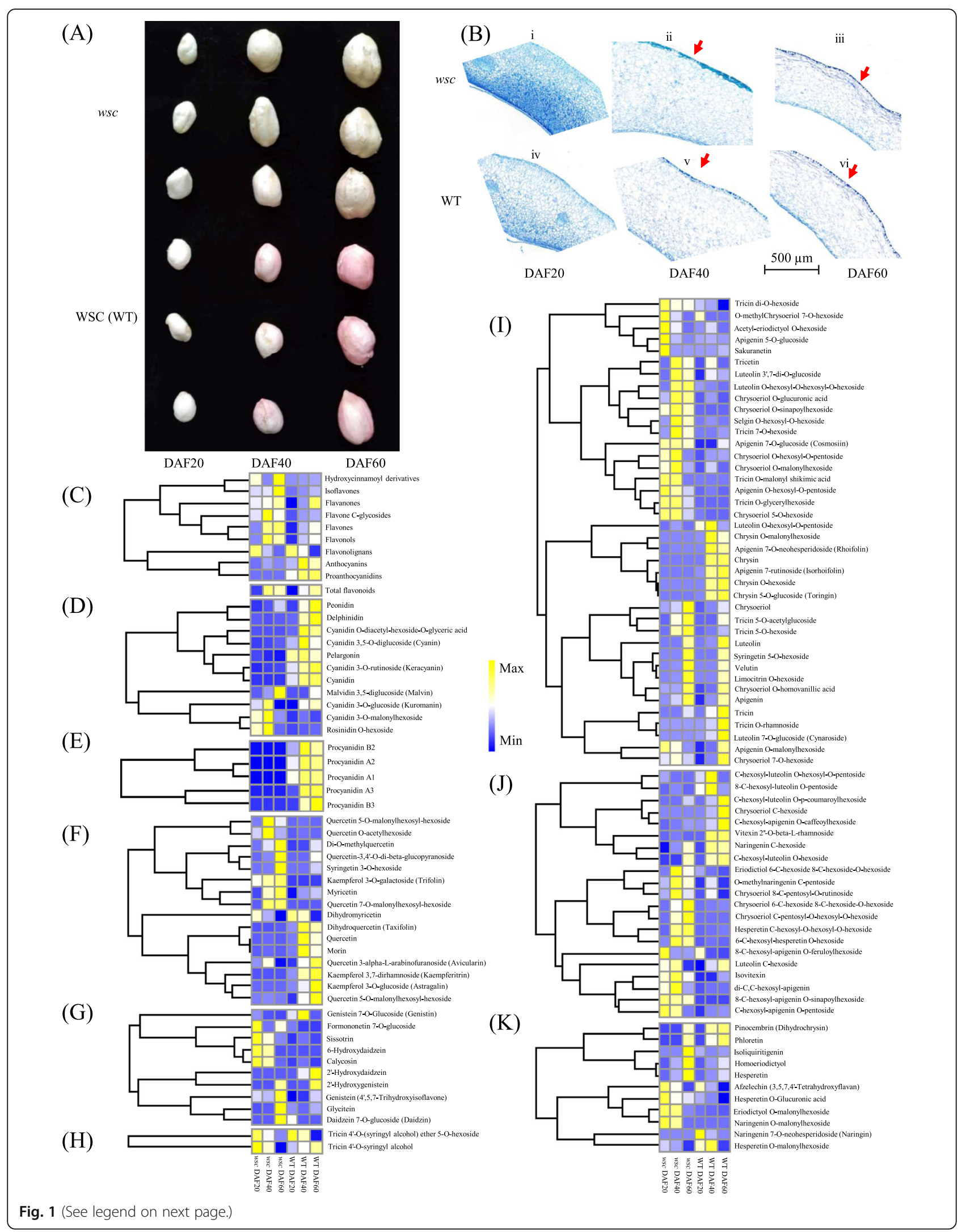


(See figure on previous page.)

Fig. 1 Flavonoids redirected in the testa of wsc. a Testa color differed between wsc and WT during development. b Staining of phenolic compounds during seed coat development in wsC and WT. c Heatmap of total flavonoids and each component contents of total flavonoids between ws and WT. d Heatmap of anthocyanins contents between wsC and WT. e Heatmap of proanthocyanidins contents between wsc and WT. $\mathbf{f}$ Heatmap of flavonols contents between ws and WT. $\mathbf{g}$ Heatmap of isoflavonols contents between wsc and WT. $\mathbf{h}$ Heatmap of flavonolignans contents between wsc and WT. i Heatmap of flavones contents between wsC and WT. $\mathbf{j}$ Heatmap of flavone C glycoside contents between wsc and WT. k Heatmap of flavanone contents between wsc and WT. The metabolite contents were scaled using Z-score of peak area (mean value of three biological replications) in the heatmap.

while developing; flavonoid content peaked at DAF40 in wsc compared with WT samples at DAF60 (Fig. 1c). Anthocyanins, proanthocyanidins, and flavonols, all the dominate flavonoids, were enhanced throughout development except flavonolignan. The anthocyanins detected in this study included cyanidin, delphinidin, malvidin, pelargonidin, peonidin, rosinidin, and derivatives, data showed that peonidin, delphinidin, cyanidin O-diacetyl-hexoside-Oglyceric acid, cyanidin 3,5-O-diglucoside (cyanin), pelargonin, cyanidin 3-O-rutinoside (keracyanin), and cyanidin levels all increased throughout the development of testae in WT while remained at very low stable levels in wsc. Levels of the four glycosylated anthocyanins [i.e., malvidin 3,5-diglucoside (Malvin), cyanidin 3-O-glucoside (Kuromanin), cyanidin 3-O-malonylhexoside, and rosinidin $\mathrm{O}-$ hexoside] all increased in wsc during the two early developmental stages and then decreased to equal amounts in the WT at DAF60 (Fig. 1d), only malvin keeps the increasing trend in wsc. At the same time, procyanidin A1, procyanidin A2, procyanidin A3, procyanidin B1, and procyanidin $\mathrm{B} 2$ all occurred at higher levels in WT samples, especially at DAF40 and DAF60, while procyanidin contents in wsc remained three orders of magnitude lower (Fig. 1e). The content of flavonols was also redirected over the course of this experiment between wsc and WT, and eight of these were up-regulated while nine were downregulated in wsc (Fig. 1f). Indeed, both the majority and total amounts of isoflavones were enhanced in wsc (Fig. 1g), while flavonolignan volumes did not change significantly between WT and wsc (Fig. 1h). Flavones were also redirected between wsc and WT; the total contents of these compounds were surprisingly enhanced in wsc (Fig. 1c, i). A total of 13 flavone $\mathrm{C}$ glycosides were upregulated in this analysis while eight were down-regulated in wsc although total contents obviously increased overall (Fig. 1c and j). Considerable increases in four flavanones were also seen during the two early developmental stages, three were significantly strengthen at DAF60, while two of the other four differentiated flavanones had declined at DAF60 as well as one at DAF20 and DAF 40, respectively (Fig. 1k).

An untargeted lipid metabolome analysis was also performed as part of this study identifying 6424 differential known metabolites (Additional file 2), KEGG enrichment analysis of differentiated metabolites (Additional file 2) validated the enrichment of flavonols and also showed that isoflavonoid biosynthesis between wsc and WT remained quite consistent across the flavonoid metabolome. Differential over-representation was also revealed in several metabolic pathways, including brassinosteroid, cutin, suberin, and wax biosynthesis, as well as arginine, proline, starch, sucrose, and galactose metabolism (Additional file 2).

A seed metabolome analysis was performed to investigate whether, or not, wsc influence embryo nutrient quality. A total of 439 metabolics were identified at differential levels within embryos of wsc and WT (Additional file 3) with the top five enriched pathways recorded as metabolic pathways, biosynthesis of secondary metabolites, isoflavonoid biosynthesis, flavonoid biosynthesis, and flavone and flavonol biosynthesis (Additional file 4A). Particularly, all differential metabolites from isoflavonoid biosynthesis were up-regulated during this analysis (Additional file 4A). At the same time, five of the six metabolites from flavone and flavonol biosynthesis were enhanced, and the contents of 3,7Di-O-methylquercetin, quercetin 3-O-[beta-D-xylosyl(1- > 2)-beta-D-glucoside], and kaempferide in wsc rose to more than five times those seen in WT (Additional file 3). All metabolites involved in phenylpropanoid biosynthesis and phenylalanine metabolism declined at different levels (Additional file 3), while both the two involved in cutin, suberin, and wax biosynthesis were down-regulated in wsc (Additional file 4A). The results of this analysis are consistent with those from testae flavonoid metabolomes. Testae play crucial role in transporting, as the main energy storage form, fatty acid contents in wsc and WT were tested. Results reveal that almost all fatty acid components together with the total fatty acid contents decreased over the course of this study between 5.47-19.41\% (Additional file 4B).

\section{Global transcriptome analysis reveals the involvement of multiple biological processes during testa development}

In order to identify the genes that underlie the formation of white testa phenotype, RNA-seq were conducted on both wsc and WT samples collected at DAF20, DAF40, and DAF60. Fold change $\geq 2$ and stringent FDR value $\leq 0.001$ were used as thresholds to identify DEGs. Results revealed a total of 17,428 DEGs between wsc and WT 


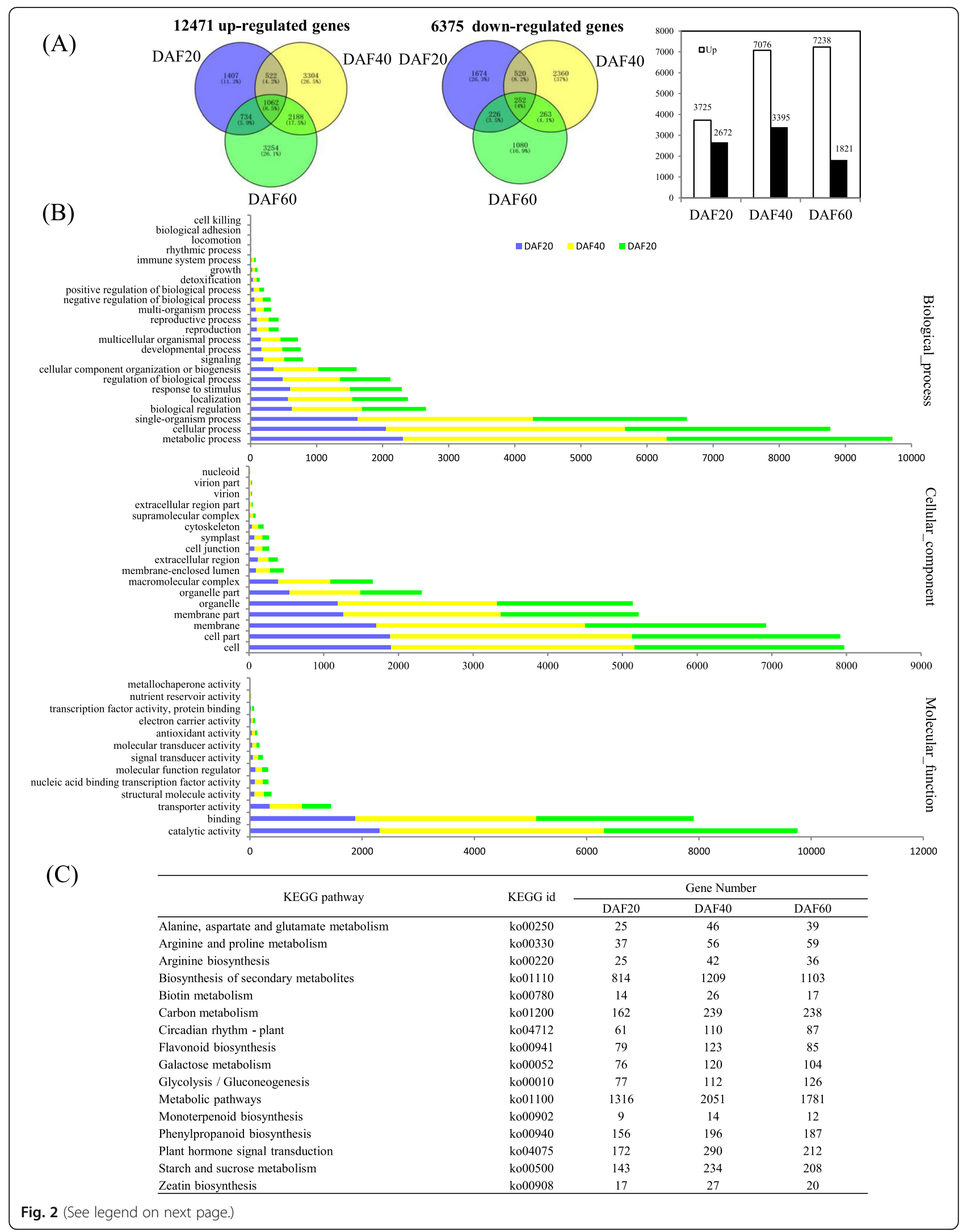


(See figure on previous page.)

Fig. 2 Reprogrammed gene expression in white peanut testae. a Venn diagrams displaying overlap between DEGs identified in peanut testae at DAF20, DAF40, and DAF60. The numbers in parentheses showed percentages with respect to the total upregulated and downregulated genes. $\mathbf{b}$ Selected GO terms enriched among DEGs identified in peanut testae of wsC compare with wild types for indicated times. Color panels highlight the three developmental stages assessed in this study. c KEGG pathway enrichment analysis among DEGs identified in peanut testae of wsC compared with WT for designated DAF period

(Fig. 2a; Additional file 5). A total of 6397DEGs, 10,471 DEGs, and 9059 DEGs were identified at DAF20, DAF40, and DAF60, respectively (Fig. 2a). Up-regulated genes (3725) presented at DAF20 were significantly more than down-regulated ones (2672), while the number of up-regulated genes (7036) at DAF40 was much more than those down-regulated (3395). 7238 upregulated genes and 1821 down-regulated ones were uncovered at DAF60 (Fig. 2a).

To analyze the functions of the DEGs between wsc and WT, a GO analysis was performed using GOseq method in Blast2GO. Data revealed that metabolic process, cellular process, single-organism process, biological regulation, and localization were all dominant biological process categories (Fig. 2b, Additional file 6). In terms of $\mathrm{GO}$ terms relating to cellular component, most DEGs were correlated with five major biological processes, including cell as well as cell part, membrane, membrane part, and organelle (Fig. 2b, Additional file 6). Catalytic activity, binding, transporter activity, structural molecule activity, and nucleic acid binding transcription factor activity were the top categories annotated for the molecular function (Fig. 2b, Additional file 6).

Further, 132, 135, and 134 metabolic pathways of the DEGs associated with the three different developmental stages of the two lines were uncovered by KEGG pathway analysis, respectively. A total list of the metabolic pathways identified in this study is presented in Additional file 7, alongside the top 16 common metabolic and biological pathways identified in the wsc compared with WT (Fig. 2c). These results revealed that metabolic pathways were the most enriched, followed by secondary metabolite biosynthesis, plant hormone signal transduction, carbon metabolism, endocytosis, starch and sucrose metabolism, and amino acid biosynthesis (Additional file 7).

\section{qPCR verification of wsc and WT DEGs}

To validate the repeatability and reproducibility of gene expression data obtained by RNA-seq, we performed biologically independent qRT-PCR to test the expression level of 17 genes with FPKM values $\geq 2$. The genespecific primer pairs designed with the Primer Express 3.0 software used for this analysis are listed in Additional file 8 . The linear regression analysis revealed a well correlation coefficient $(R)$ of 0.84 , indicating a strong relationship between the expression data revealed by RNA-Seq and abundances assayed using qRT-PCR (Additional file 9).

\section{WSC mutation influences flavonoid metabolic pathways}

Flavonoid components determine the color of the peanut testa [21-23]. Flavonoid biosynthesis pathway genes were searched based on their KO identifications within the KEGG database as well as synonyms identified in combined with functional annotations, this enabled the identification of 433 DEGs in flavonoid metabolic pathways (Table 1). The genes involved in the three secondary metabolic pathways (i.e., flavonoid, anthocyanin, and flavones/flavonol biosynthesis) related to pigmentation were analyzed in this study using testa transcripts, the core genes in these pathways were studied in detail, and results demonstrated that most exhibited significant changes in expression levels over the course of this analysis. Indeed, regardless of whether these were EBGs (e.g., $C H I$ ) or LBGs (e.g., ANS, UFGT), all exhibited higher transcript abundances within WT compared to wsc with the exception of 4-coumarate: CoA ligase genes and their flavonol synthase counterparts (Fig. 3). As a branch point in flavonoid biosynthesis, the dihydroflavonols are the intermediates in the production of both the colourless flavonols and the colored anthocyanins through FLS and DFR, respectively. Anthocyanins contents were decreased in wsc, which corresponds to lower level expression of $D F R$ genes. While, myricetins and kaempferols contents were increased in wsc, this consistent with FLS expression (Fig. 3). Combining the transcriptome and metabolome information, it could be extrapolated that DFR might be the target gene for the loss of pink color in wsc. The competition between FLS and DFR for common dihydroflavonols substrates might obviously block anthocyanins synthesis and leading to the enhanced production of flavonols such as myricetin and kaempferol, which shift the flavonol: anthocyanin ratio in $w s c$.

\section{Suberin in wsc testa epidermis}

Suberin and its associated waxes play important roles in plant development [24]. As flavonoids in the testa of wsc changed greatly over the course of this analysis, KEGG pathway involved in cutin, suberin, and wax biosynthesis were further analyzed in this study. Data indicated that most cutin and suberin synthesis pathway genes showed significantly higher levels of gene transcripts in wsc 
Table 1 Flavonoid pathway genes related to testa pigmentation of peanut

\begin{tabular}{|c|c|c|c|c|c|c|c|}
\hline Function & Gene & Enzyme & KO id (EC.no) & No.All & $\begin{array}{l}\text { DEGs in } \\
\text { DAF20 }\end{array}$ & $\begin{array}{l}\text { DEGs in } \\
\text { DAF40 }\end{array}$ & $\begin{array}{l}\text { DEGs in } \\
\text { DAF60 }\end{array}$ \\
\hline & PAL & Phenylalanine ammonia-lyase & K10775 & 14 & 2 & 5 & 0 \\
\hline & $\mathrm{C} 4 \mathrm{H}$ & Trans-cinnamate 4-monooxygenase & K00487 & 6 & 5 & 2 & 3 \\
\hline & $4 C L$ & 4-coumarate--CoA ligase & K01904 & 45 & 8 & 15 & 6 \\
\hline \multirow[t]{7}{*}{ Anthocyanin biosynthesis } & CHS(STS) & Chalcone synthase(Stilbene synthase) & K00660 & 76 & 11 & 43 & 18 \\
\hline & $\mathrm{CHI}$ & Chalcone isomerase & K01859 & 21 & 5 & 5 & 3 \\
\hline & $\mathrm{F} 3 \mathrm{H}$ & Flavanone 3-hydroxylase & K00475 & 9 & 2 & 5 & 4 \\
\hline & $F 3^{\prime} H$ & Flavanone 3'-hydroxylase & K05280 & 11 & 0 & 1 & 0 \\
\hline & DFR & Dihydroflavonol 4-reductase & K13082 & 17 & 5 & 6 & 5 \\
\hline & ANS & Anthocyanidin synthesis & K05277 & 20 & 6 & 4 & 9 \\
\hline & UFGT & Anthocyanidin 3-O-glucosyltransgersae & K12930 & 5 & 0 & 0 & 0 \\
\hline \multirow[t]{5}{*}{ Anthocyanin modification } & UGT75C1 & Anthocyanidin 5-O-glucosyltransgersae & K13692 & 52 & 12 & 7 & 3 \\
\hline & GT1 & Anthocyanidin 5,3-O-glucosyltransgersae & K13263 & 6 & 0 & 3 & 0 \\
\hline & FLS & Flavonal synthase & K05278 & 70 & 14 & 18 & 14 \\
\hline & FOMT & Flavonol 3-O-methyltransferase & K05279 & 39 & 3 & 3 & 5 \\
\hline & UF3GT & flavonol 3-O-glucosyltransferase & K12930 & 5 & 0 & 0 & 0 \\
\hline \multirow[t]{2}{*}{ Flavanone biosynthesis } & ANR & anthocyanidin reductase & K08695 & 3 & 2 & 2 & 2 \\
\hline & $L A R$ & leucoanthocyanidin reductase & K13081 & 34 & 10 & 9 & 9 \\
\hline
\end{tabular}

(Fig. 4a and b) while the genes involved in wax synthesis exhibited no obvious regularity (Fig. 4c). Five of the six FAR gens involved in the synthesis of aromatic suberin monomers for the aliphatic domain [25] were significantly depressed at either DAF20 or DAF60, eight of 11 HHT1 DEGs were up-regulated, and all CYP86B1 genes exhibited a higher level of expression in wsc (Fig. 4a). Four out of five CYP86A4S genes were classified as DEGs while all four $H T H$ genes exhibited enhanced expression levels compare with WTs (Fig. 4a). Suberin staining revealed the presence of almost no red staining in all three developmental stages of wsc compared with the deeper red staining in WT during development (Fig. 4d).

\section{Primary metabolic differences}

Transcriptome analysis revealed that the glycolysis and gluconeogenesis pathway was differentially regulated between the wsc and WT. The expression of coding genes for the 13 enzymes catalyze the 14-step reactions in glycolysis was also enhanced to varying degrees (Additional files 10, 11). All nine DEGs in hexokinase which function as one of the three key enzymes (i.e., hexokinase, phosphofructokinase, and pyruvate kinase) within the glycolysis pathway markedly increased in expression, especially in the two later stages (Additional files 10A, 11A), while the six DEGs in phosphofructokinase exhibited higher expression levels in wsc via different patterns (Additional files 10A, 11B). Two of the 12 pyruvate kinase genes were down-regulated in the wsc, especially during the early stage at DAF20, including the highest expressed gene, Araip.VA90H. The remaining ten pyruvate kinase genes also exhibited enhanced expression in the mutant (Additional files 10A, 11C).

The products of the glycolysis pathway were then catalyzed by the pyruvate dehydrogenase complex and entered into the TCA cycle. As data revealed that 19 DEGs, 26 DEGs, and 37 DEGs were enriched in this cycle at DAF20, DAF40, and DAF60, respectively, the ten enzyme encoding genes that control this process were analyzed in detail and six were found to be differentially expressed in the wsc. Four pyruvate dehydrogenase genes were up-regulated in all three developmental stages in the wsc, while the other three were only enhanced at DAF40 (Additional files 10B, 11D). At the same time, two citrate synthase genes increased and one deceased in the wsc (Additional files 10B, 11E), while all aconitase genes were characterized by activated expression in this mutant, especially the three high expressed genes (Aradu.SOKU9, Aradu.83N8C, and Araip.SB6JF) (Additional files 10B, 11F). Isocitrate dehydrogenase expression was also universally higher in the wsc, reaching its highest level at DAF40 (Additional files 10B, 11G), while both DEGs in succinate dehydrogenase differed at DAF40 with one activated and the other one depressed (Additional files 10B, 11H). Five of the six malate dehydrogenase genes were up-regulated at either DAF40 or DAF 60 (or both), while Araip.M2TZZ was downregulated in all the three wsc developmental stages (Additional files 10B, 11I). 


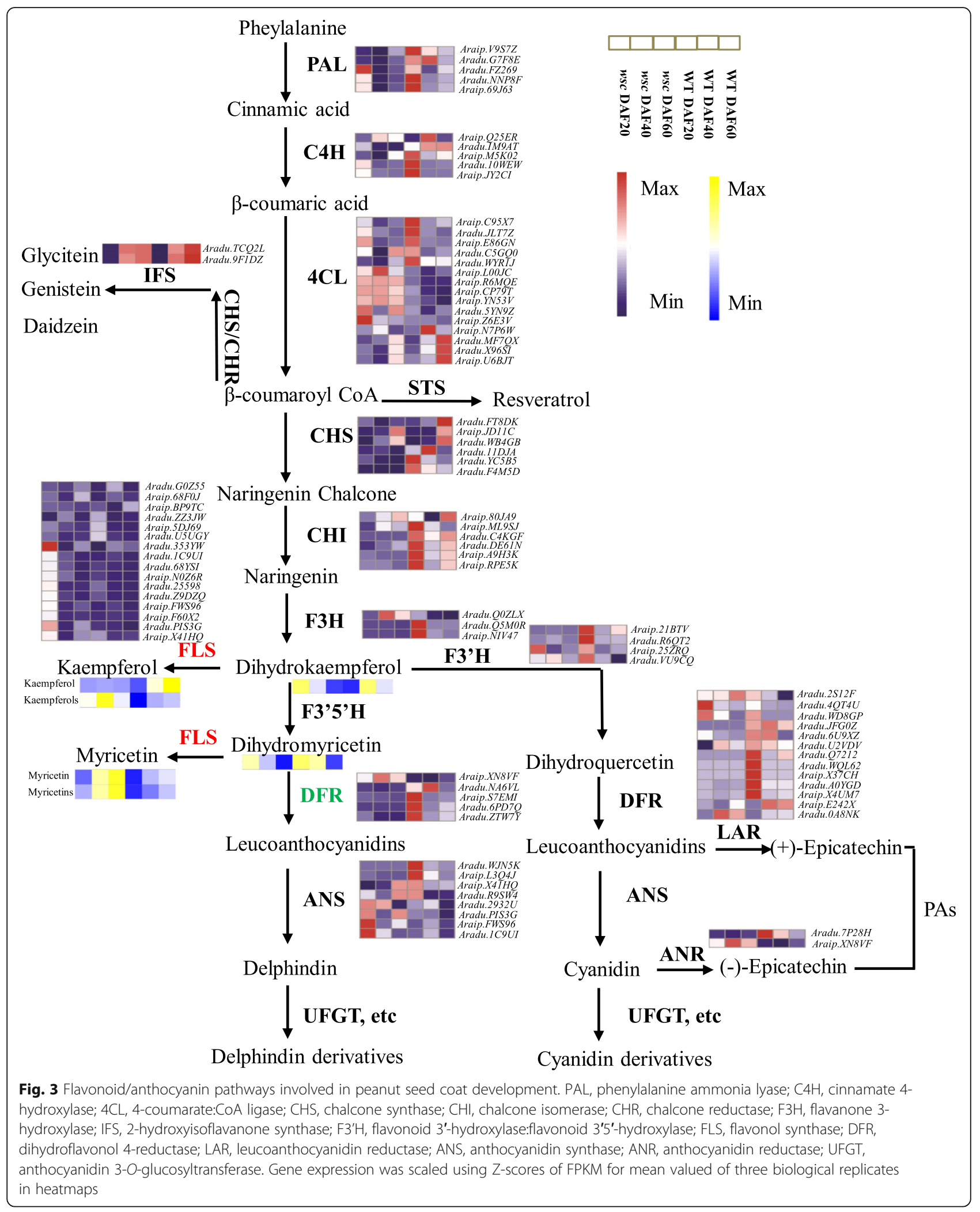




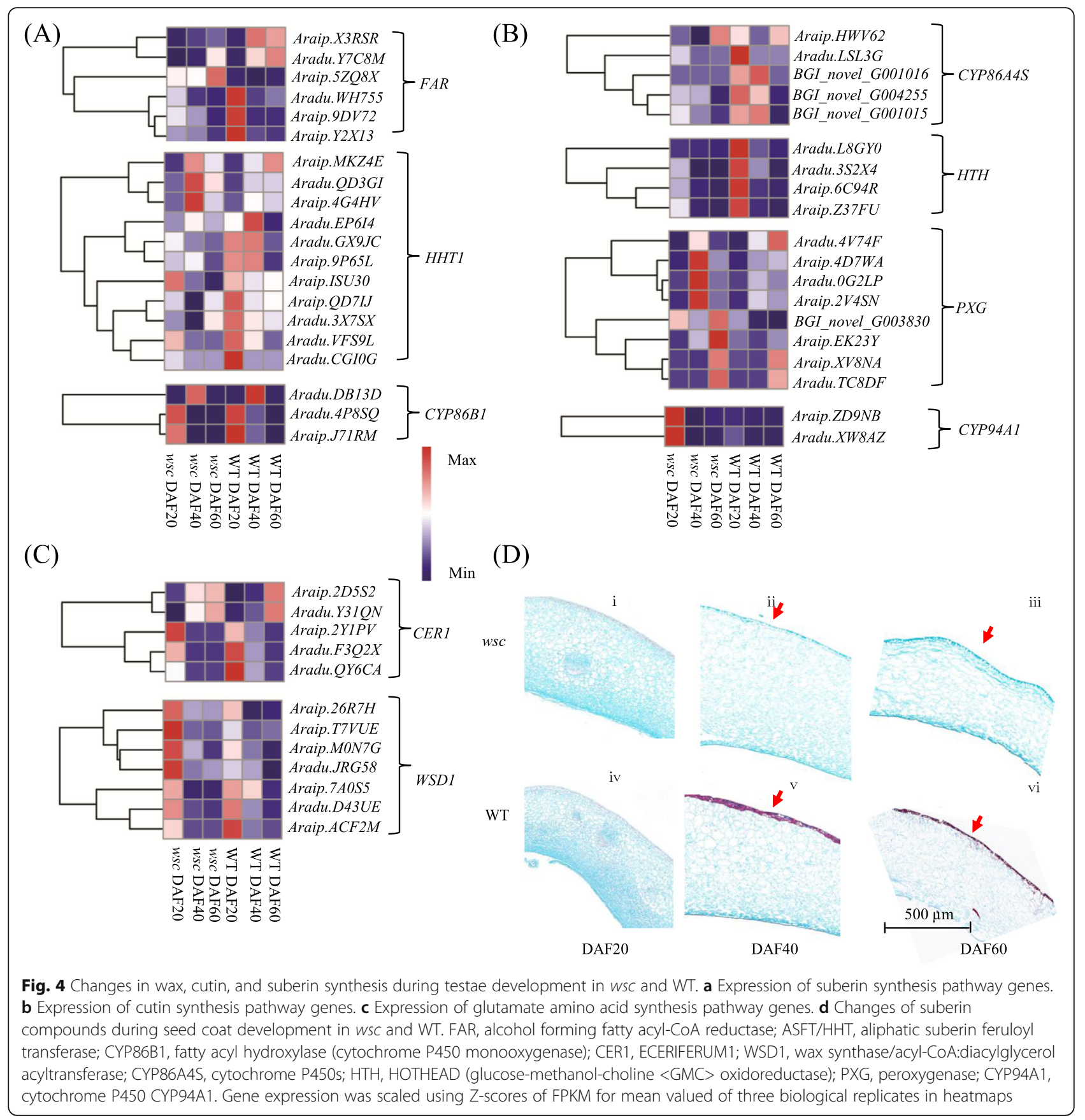

DEG investigations within amino acid metabolism pathways revealed that glutamate amino acid synthesis occurred at significantly higher expression levels within wsc (Fig. 5a). Five of P5CS (one of the two key enzyme coding gene in proline synthesis) genes were all characterized by stronger levels of expression at all three developmental stages while the other three had higher expression levels in the wsc at the two early stages. Two P5CR (another key enzyme coding gene in proline synthesis) gene were detected in differential expression; Aradu.A85Y5, was characterized by a significantly higher expression level at all three stages while its counterpart, Aradu.B47VE, was only activated at DAF20 and DAF40 (Fig. 5a). Metabolome analysis of testae revealed that the proline content in wsc was three times that of WT while the N-Carbamoylsarcosine content reached 1.8 times that level when compared with WT (Fig. 5b).

Sugars supply plants with energy and functioned in flavonoids accumulation [26, 27]. Three gene families encoding sucrose transporters have been implicated in plant sugar accumulation, including TSTs [28], SUTs [29-31], and clade III of SWEET [32-34]. We compared 


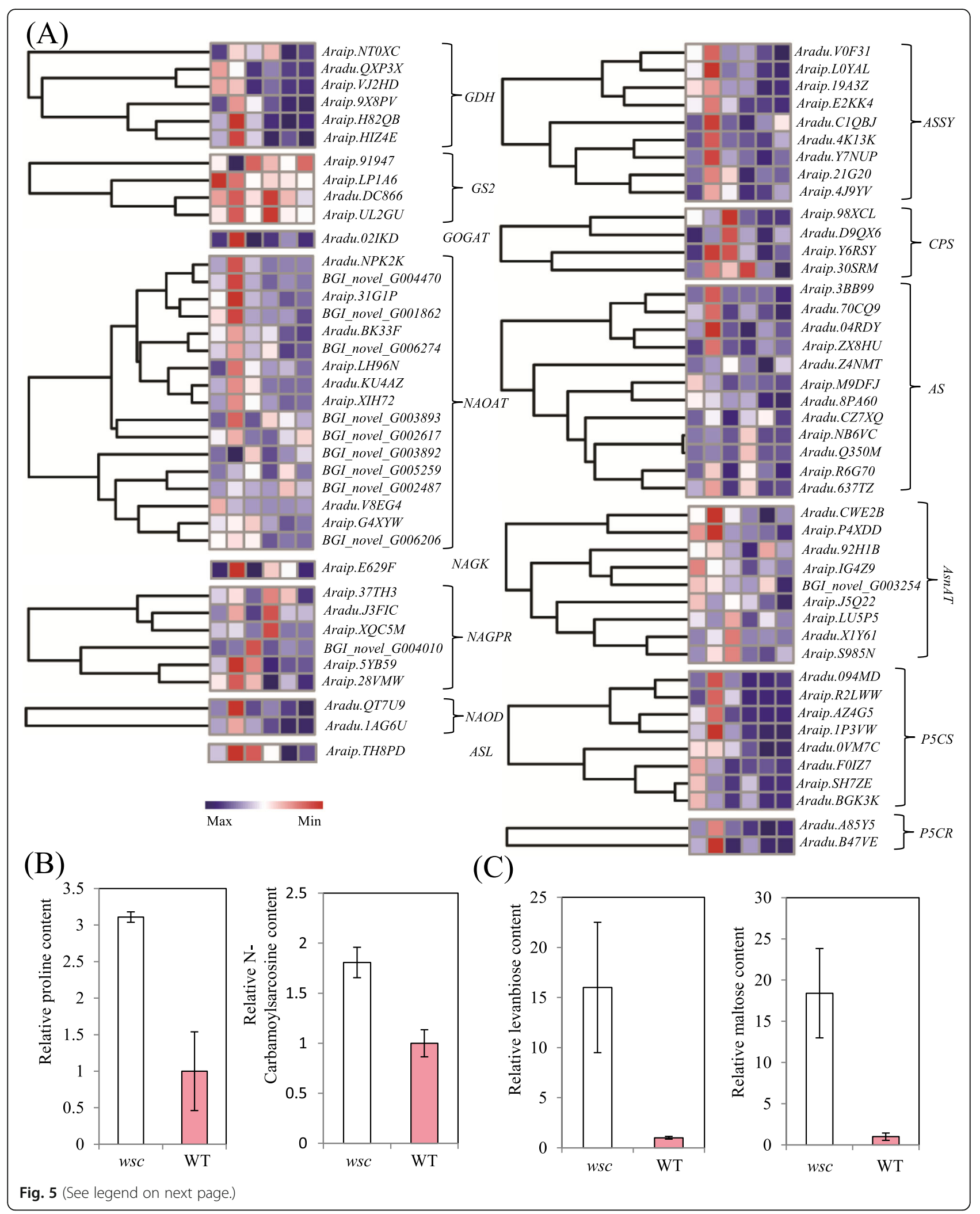




\section{(See figure on previous page.)}

Fig. 5 Expression of glutamate amino acids synthesis pathway genes and altered amino acid/sugar contents in wsC and WT. a Expression of glutamate amino acids synthesis pathway genes. b Proline and N-carbamoylsarcosine content in wsc and WT. c Levanbiose and maltose content in wsC and WT. GDH, glutamate dehydrogenase; GS2, glutamine synthetase; 2, GOGAT, glutamate synthase; NAOAT, N-acetylornithine aminotransferase; NAGK, N-acetylglutamate kinase; NAGPR, N-acetylglutamatyl-5-P reductase; NAOD, N-acetylornithine deacetylas; ASL, argininosuccinate lyase; ASSY, argininosuccinate synthase; CPS, carbamoyl phosphate synthetase; AS, asparagine synthase; AsnAT, aspartate aminotransferase; P5CS, delta-1-pyrroline-5-carboxylate synthetase; P5CR, pyrroline-5-carboxylate reductase. Gene expression was scaled using Zscores of FPKM for mean valued of three biological replicates in heatmaps

the expression patterns of these gene families between wsc and WT (Additional file 12) and found seven SUTs to occur at significantly higher levels within wsc than WT even though expression patterns were different; Aradu.M7BX0, Aradu.QA3D9, and Aradu.SQ8X7 were all owned highest levels at the early developmental stage and adjusted to a moderate level; in contrast, Araip.53MMF exhibited a higher expression level within wsc at DAF60 while the other three SUTs were characterized by consistently higher expression levels in mutants at the all three stages (Additional file 12). Nine of the 13 DEGs in SWEETs were enhanced and four decreased in expression (Additional file 12). In the case of the monosaccharide transporter, expression of the two STPs in wsc was dramatically higher than in WT (Additional file 12). The metabolome profile data elucidated that wsc testae included more than ten-fold increases in maltose and levanbiose contents compare with WT (Additional file 12C).

\section{The vital roles of phytohormones and TFs in the white testa phenotype of wsc}

Hormone and transcription factors have both been reported to perform functions in flavonoid accumulation $[15,35]$. Genes involved in hormone synthesis and signal transductions were compared in this study. Results revealed the presence of five DEGs involved in the BR synthesis pathway, of which three BR6OX1 were upregulated either at DAF20 or at DAF40 and DAF60; in contrast, the two $B A S 1$ genes which reduce the level of active BRs were significantly down-regulated in wsc at DAF60 (Additional file 13A). Analysis of the testae metabolome uncovered 16 metabolites within the BR biosynthesis pathway, and all of them were up-regulated (Additional file 2). At the same time, 16 DEGs involved the GA synthesis pathway including $K A O$ (3), GA2ox (3), GA3ox (1), and GA20ox (9) were identified in this analysis; the majority of these genes were up-regulated in $w s c$ with the exception of Aradu.BUI3V, Araip.KVM2C, and Aradu.U5UGY that all exhibit opposite expression patterns (Additional file 13B). Further, testae metabolome analysis also supported the enhancement of GA synthesis by identifying three pathway products that increased in content within wsc (Additional file 2). While DEGs involved in JA biosynthesis included two PLA1 (i.e., BGI_novel_G004010 and Aradu.Y096Z), one AOC
(Aradu.H2RVW), four OPR (i.e., Aradu.FV6YV, Araip.F1ZZD, Aradu.OCOYF, and Araip.22RGE), and three MFP (i.e., Araip.UPC07, Araip.M6X0I, and Araip.60KBF) were all enhanced in wsc (Additional file 13C). These expression and metabolome data taken together therefore supported the enhancement of BR, GA, and JA synthesis pathways.

A series of DEGs involved in seven hormone signal transduction pathways were also identified in this study, including nine in ABA, 12 in AUX, five in BR, ten in CTK, seven in GA, ten in JA, and four in SA (Additional file 14). Seven ABA signaling DEGs were enhanced, including SRK2 gene Aradu.SKS95 that exhibited the highest FPKM value (Additional file 14A). The vast majority of AUX signaling pathway genes increased in expression in wsc; only two deviated from this pattern, Araip.TU273 and Araip.60Q0E, both of which exhibited obvious decrease in mutants at DAF20 (Additional file 14B). Further, four out of five DEGs involved in BR signaling were highlighted in wsc (Additional file 14C); and nine of the ten DEGs involved in JA signal pathway were up-regulated and all of them were MYC2 factors (Additional file 14D). Expression patterns and FPKM values of DEGs in hormone signal transduction pathways provided collective evidence of ABA, AUX, JA, and BR signaling enhancements (Additional file 14).

Flavonoid production is transcriptionally regulated by MYB factors and the MBW complex. A total of 24 R2R3MYB (nine up and 15 down), 26 bHLH (17 up and nine down), and 21 WD40 (19 up and two down) genes were identified in this analysis (Additional file 15) including homologs (Aradu.CA8XJ and Araip.MHR6K) of AtTT8 which enables strong, cell-specific accumulation of flavonoids in Arabidopsis thaliana [36] and homologs of AtMYB5 (i.e., Aradu.JK51Z and Aradu.WZFOO) control outer seed coat differentiation alongside TTG1 and TT2 [37].

\section{White testa phenotype candidate genes revealed by multi-omics analysis}

In order to identify candidate genes controlling the white testa mutant phenotype in peanut, we analyzed DEGs in common between WT and wsc at the three different developmental stages. This analysis resulted in the identification of 1646 unigenes (Fig. 2 and 
Additional file 16). Observations across whole growth stages and tissues revealed no obvious differences between wsc and WT with the exception of the seed phenotype (Additional file 17), therefore WSC was identified as a seed-specific gene. Previously published data [38] was interrogated to identify seed specific expressed common DEGs between wsc and WT which resulted in 86 candidates genes for the testa phenotype (Additional file 18). The wsc was identified in the M3 generation of the mutant population while the segregation ratio of the mutant line between WT and wsc was nearly 3:1 (156:49). Combining seed specific expressed common DEGs with the results of the genetic analysis revealed the putative candidate genes Araip.M7RY3 (CSN1), Aradu.R8PMF (MYB) and Araip.MHR6K (bHLH) (Additional file 18). The FPKM value of the Araip.M7RY3 gene in the WT seed coat decreased from 2.15 (DAF20) to 1.15 (DAF40) before falling further to 0.19 , while the value for this gene in wsc increased from 7.55 (DAF20) to 12.21 (DAF40) and then rose further to 12.84 (DAF60). It is annotated that Araip.M7RY3 is homolog of a COP9 signalosome complex subunit 1 (CSN1) encoding gene. Previous studies have revealed that several subunits of the COP9 signalosome complex are involved in regulating flavonoids and that phenylalanine metabolism further regulates proanthocyanidin biosynthesis [39-41]. Aradu.R8PMF and Araip.MHR6K are components of MBW complex which are widely reported function in flavonoids metabolism (Li, 2014). To investigate the differences of the sequences of the three candidate genes between the WT and the mutant plants, we checked SNPs called from the transcriptome data and verified the SNPs by the direct sequencing of the PCR products. As a result, we discovered one SNP in the DNA binding domain of bHLH-MYC_N (pfam14215) of Araip.MHR6K (Araip.B06_2335496 $6^{[\mathrm{A} / \mathrm{G}]}$ ) leadting to a $50^{\mathrm{T}}$ to $50^{\mathrm{I}}$ amino acid change which was confirmed by sequencing (Additional file 19). The information that these genes cause a white seed color phenotype require further confirmation in future functional genomics studies.

\section{Discussion}

Appropriate pigmentation determines the appearance and nutrient quality of peanuts. Complex transcriptome, metabolome regulation, and cytological changes therefore suggested that secondary metabolism pathways like flavonoid biosynthesis as well as primary metabolism pathways like glycolysis and gluconeogenesis represented clear differences between $w s c$ and WT. Flavonoid synthesis-related genes as well as their counterparts involved in key enzyme coding genes in glycolysis and the TCA cycle, glutamate amino acid synthesis pathway genes, and sugar synthetase and transporter genes differentially expressed between wsc and WT. These pathways might well be regulated by upstream phytohormone signals (AUX, BR, and JA) and transcription factors (MYB, bHLH, and WD40). The results provided informative clues that augment understanding of the regulatory network that underlies testa coloration and will therefore contribute to the genetic breeding of ideal quality peanuts.

\section{Multilevel regulation of the WSC gene}

The gene enrichment analysis reported here revealed that 23,17 , and 13 biological processes, cellular component and molecular function mainly associated with metabolism and environmental information processing differentially altered (Fig. $2 \mathrm{~b}$ and Additional file 6). Genes associated with metabolic process comprised 36.08, 38.09, and 37.71\% (i.e., 2308/6397, 3988/10471, and 3416/9059) of total DEG numbers (Additional files 5 and 6), while metabolic pathways, the biosynthesis of secondary metabolites, plant hormone signal transduction, and carbon metabolism were the major biological processes influenced by wsc (Fig. 2b). Genes associated with metabolism exhibited a mixed trend, while hormone responses and biosynthesis processes were effectively up-regulated in wsc.

The DEGs detected in this study were classified into 16 co-expression modules, each of which contains representatives that harbor similar expression patterns and might therefore have parallel functions (Fig. 6a, b). KEGG enrichment revealed that 14 co-expressed DEG modules were all enriched in flavonoid biosynthesis, plant hormone synthesis, plant signal transduction, and carbon metabolism. Multi-omics analysis implying the competitive expression of FLS and DFR genes resulted in the redirect of flavonols and anthocyanin accumulations. Additional emphasis was then placed on modules containing both FLS and DFR genes. Surprisingly, we found FLS genes were co-expressed with MYB genes (Araip.2H669), WD40 (Aradu.F5YFV), AOC (Araip.Q7E6I, Aradu.T8ILN, and key enzymes coding genes in the JA synthesis pathway), JAZ (Aradu.X4R7M and Aradu.GFT6J), MYC2 (Aradu.DSN52, Aradu.B7RDX, and Araip.LGM59) and IFS (Araip.RHC93 and Araip.E734B), indicating the regulation of the FLS genes by MBW complex and the JA signal pathway (Fig. 6c). At the same time, DFR genes were co-expressed with $\mathrm{CHS}$ (Aradu.F4M5D), F3H (Araip.NIV47 and Aradu.Q5MOR), ANR (Araip.8TB4E) and LAR (Araip.X37CH and Ara$d u$.WQL62) genes (Fig. 6d). It has been reported that both anthocyanin and procyanidine synthesis is regulated by the MBW complex in Zea mays, Antirrhinum majus, Petunia hybrid, and A. thaliana [37, 42-46]; although the results of this analysis are inconsistent with above studies, they are nevertheless consistent with the 


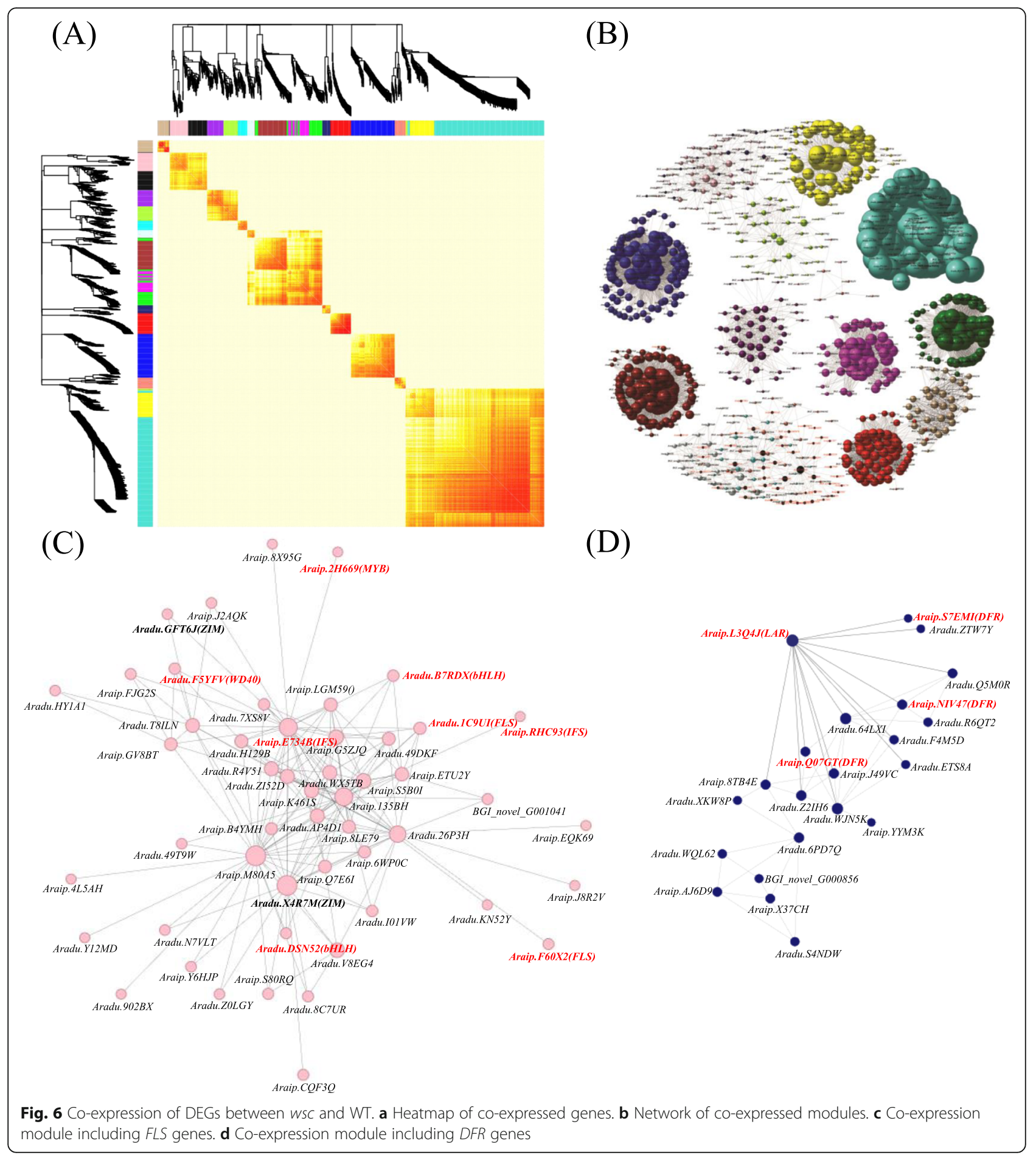

results of peanut pigment regulation research which has shown that anthocyanin biosynthesis is mainly regulated by AtMYB111 homologs (i.e., c35101_g4 and c37398_g2) through EBGs instead of through the MBW complex via LBGs. The results of this study therefore highlight the distinct regulation patterns in peanut testa pigments, possibly from the trait of flower aerially and producing pods underground.
WSC redirects the phenylpropanoid metabolic flux in peanut testa

Flavonoids are secondary metabolites that accumulate in plants and promote seed and pollen dispersal by contributing to the formation of color in fruits and flowers [47]. Indeed, when the wsc was first observed, it was thought that flavonoid content must be markedly decreased 
compared with WT for the white color, however, that TBO staining of transverse developing testae sections present no huge differences in wsc seeds stained colors while metabolome analysis revealed that isoflavones, flavanones,flavones, flavone $\mathrm{C}$-glycosides, flavonols were all significantly increased while anthocyanins and proanthocyanidins contents markedly decreased. Flavonoid Cglycosides have recently been shown to exhibit significant antioxidant activity as well as anticancer and antitumor potential, hepatoprotective, anti-inflammatory, antidiabetes, antiviral, antibacterial, and antifungal activities as well as other biological effects [48]. It appears that Cglycosylflavonoids in most cases exhibit higher antioxidant and anti-diabetes potential than their corresponding O-glycosyl flavonoids and aglycones [48]. Redirected in flavonoid components, especially the significantly higher flavone $\mathrm{C}$-glycosides and flavonols contents, combined with a very high yield potential imply broad and extensive prospective applications for wsc.

\section{The crucial roles of phytohormones in the formation of a white testa phenotype}

Previous studies have elucidated the role of phytohormones in the regulation of flavonoid pathways. MeJA is a phytohormone which plays a key role in plant growth as well as in many physiological and biochemical processes [49]. This compound has been used to stimulate secondary metabolite production in numerous plant species. MeJA enhances antioxidant activity and flavonoid content in blackberries as well as strawberries and olive fruits [50-52]. JA synthesis in the $\alpha$-linolenic acid pathway was significantly up-regulated in both transcriptomes (i.e, all ten DEGs in the JA synthesis pathway) and metabolomes (i.e., all 16 differential metabolites were up-regulated) data sets in wsc (Additional file 13C). JAZs interacted with COI1 and then were degraded through the $26 \mathrm{~S}$ proteasome to release the downstream transcription factors such as MYC2 to activate the JA responses [53]. In this study, nine of 10 DEGs (MYC2 genes) were up-regulated in wsc. Two MYC2 genes correlated with FLS, IFS, and MSW complex were identified by the gene co-expression analysis. MYC2 modulates biosynthesis of indole glucosinolates and tryptophan, resistance to necrotrophic pathogens (e.g., B. cinerea), and the expression of ERF1, ORA59, and PDF1.2 by regulating JA-dependent responses [15, 53-59]. The multiple functions of MYC2 might be one reason for flavonoid reprogramming in wsc.

The biosynthesis of brassinolide from campesterol occurs via two alternative routes in Arabidopsis, the first

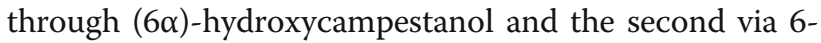
deoxycathasterone. The enzymes identified in Arabidopsis include DWF4, DET2, CPD, BR6OX, and BAS1 [60]. The members of one key gene family, BR6OX1, were up- regulated more than two-fold, while the negative regulators of BR biosynthesis, two BAS1 genes, were depressed in mutants. The expression levels of several key BR signaling and response genes, including BRI1 (Araip.0ZF73, BGI_novel_G003679), BSK (Aradu.NG7HH), and BAK1 (BGI_novel_G006285) were up-regulated by more than two-fold. It is well known that BR signaling inhibits BR biosynthesis through BES1 and BZR1 inhibition of the expression of DWF4, CPD and other biosynthesis genes [61-64]. This might be one reason why obvious BR synthesis enhancements cannot be determined.

Recent studies have also shown that IAA directly interacts with the F-box protein TIR1 and promotes the degradation of Aux/IAA transcriptional repressors to activate diverse auxin responsive genes. The results of this analysis revealed that AUX synthesis was not influenced in the wsc while signal transduction was obviously enhanced. The highest expressed AUX1 gene, Araip.TU273, was up-regulated in DAF20 while almost all AUX response genes were induced in the wsc. It has been suggested that AUX production in the endosperm drives seed coat development while fertilization of the central cell results in the production of this compound and most likely its export to maternal tissues; this process drives seed coat development by removing PcG function [65]. Activated AUX signaling might be due to strengthened AUX synthesis in the endosperm or transport from endosperm to the testa.

This study describes a peanut mutant with a white testa that contains higher levels of flavonols and flavone Cglycosides and might have a number of promising prospective applications. AUX, BR, JA, carbon metabolic and flavonoid metabolic pathways all varied between wsc and WT and so were selected as important candidates for the generation of pink testae pigmentation (Additional file 20). It is possible that the AUX signal pathway alongside BR and JA synthesis and signaling pathways cooperatively interact to modulate flavonoid synthesis and carbon metabolic pathway-related genes to influence primary metabolism and redirect the accumulation of flavonoids. Three candidate WSC gene [Araip.M7RY3 (CSN1), Aradu.R8PMF (MYB) and Araip.MHR6K (bHLH)]controls the expression of $F L S$ counterparts by regulating hormone signaling and MBW complexes were identified; in turn, this enhance the accumulation of flavonols and negatively controls transcripts of DFR genes resulting in declines in anthocyanins and PA contents (Fig. 7). The SNP in Araip.MHR6K (Araip.B06_2335496 $6^{[\mathrm{A} / \mathrm{G}]}$ ) located in the DNA binding domain which might changes its DNA binding ability with the downstream genes (especially the flavonoid synthesis pathway genes) and leads to the white testa phenotype. The SNP could be deployed in genomicsassisted breeding to develop peanut varieties with white testa. 


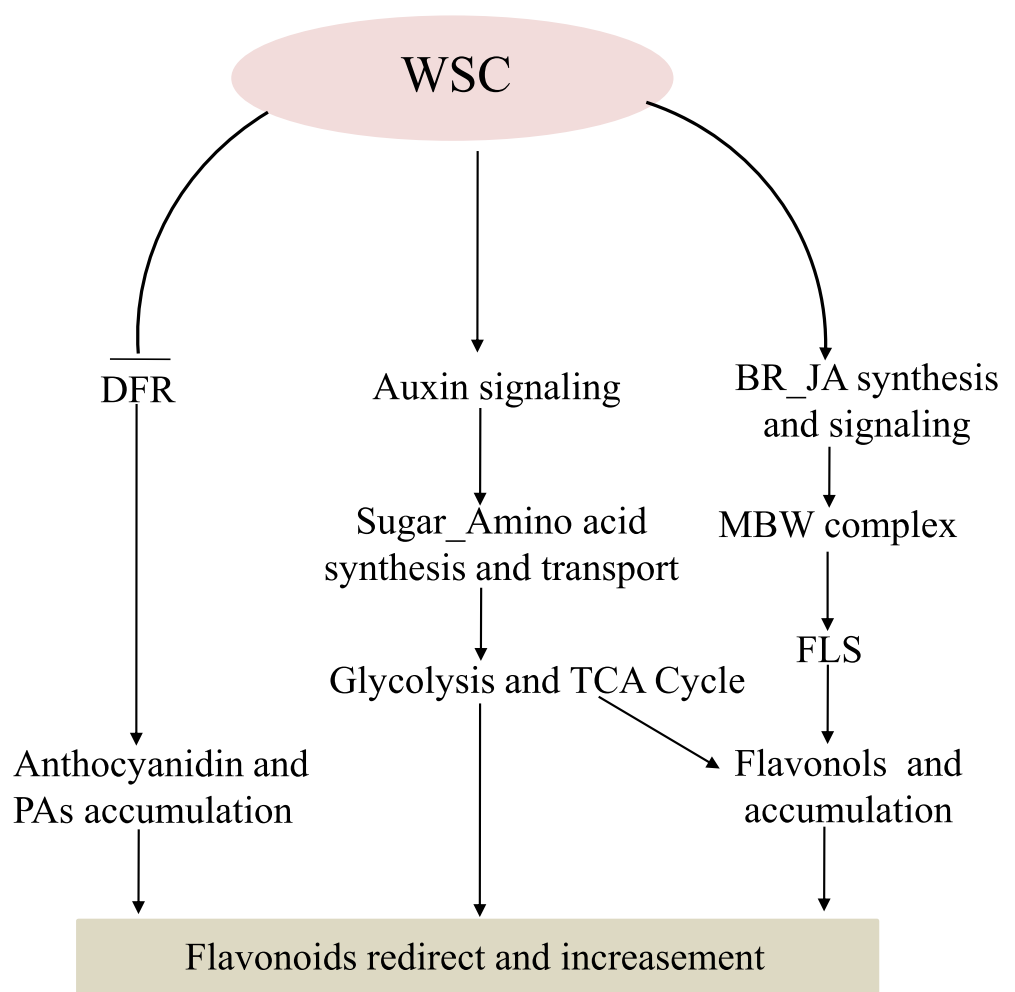

Fig. 7 Model to show the mechanism by which WSC regulates the reprogramming of flavonoids in peanut. This model involves WSC as a key factor that positively regulates flavonol and JA biosynthesis and negatively regulates anthocyanidin and PA accumulation. In addition to flavonoids biosynthesis genes, WSC also regulates the expression of several genes associated with auxin signaling, sugar, and amino acid metabolism. This model shows that WSC is an integrator of primary and secondary metabolism

\section{Conclusions}

This study describes a peanut mutant with a white testa that contains higher levels of flavonols and flavone Cglycosides and might have a number of promising prospective applications. Metabolome analysis revealed that isoflavones, flavanones, flavones, flavone C-glycosides, flavonols were all significantly increased while anthocyanins and proanthocyanidins contents markedly decreased. AUX, BR, JA, carbon metabolic and flavonoid metabolic pathways varied between wsc and WT and so were selected as important candidates for the generation of white testa pigmentation. It is possible that the AUX signal pathway alongside BR and JA synthesis and signaling pathways coordinate their interaction with modulate flavonoid synthesis and carbon metabolic pathwayrelated genes to influence primary metabolism and redirect the accumulation of flavonoids. The competition between FLS and DFR modulated by hormone synthesis and signaling as well as the MBW complex might be the key molecular controlling node regulating the white testa phenotype. Combining tissue expression patterns, genetic analyses, and the annotation of common DEGs for these three stages revealed that three testa specific expressed candidate genes, Araip.M7RY3, Aradu.R8PMF and Araip.MHR6K were likely responsible for the white testa phenotype. These data corroborate the presence of an interactive relationship between the factors responsible for white testa in wsc.

\section{Methods}

\section{Plant materials and treatments}

The wsc mutant utilized in this study was isolated from a gradient ${ }^{60} \mathrm{Co}\left(100 \mathrm{~Gy}, 40 \mathrm{~Gy} \cdot \mathrm{h}^{-1}\right)$ mutated population (3446 lines) originated by Dr. Liyun Wan from a high yield, high oil content cultivar Zhonghua 16 Cultivated by the Oil Crops Research Institute, Chinese Academy of Agricultural Sciences (OCRI-CAAS). All plants were planted within the experimental plot at OCRI-CAAS in Wuhan, China. Wild type (WT) and wsc (M7 generation) individuals were planted in parallel and seed coat samples were collected in 2017 at 20 days after flowering (20DAF), 40DAF, and 60DAF from six different plants. Three biological replicates were performed in each case and the testae separated from each sample seed were sliced. WT and wsc testae samples were then rapidly frozen in liquid nitrogen and stored at $-80^{\circ} \mathrm{C}$. Total RNA was isolated from seeds using a RNA extraction kit 
Qiagen RNeasy Plant Mini kit (QIAGEN Inc.121 Valencia, CA) following the manufacturer's protocol.

\section{Tissue preparation and light microscopy observations}

Tissue preparation was carried out as described before according to Wan et al. [23]. Sections of the tissue were stained with $\mathrm{TBO}$ and safranin $\mathrm{O} /$ fast green to reveal polyphenols and suberin, respectively [66]; these were then observed using a Nikon ECLIPSE TI-SR microscope (Nikon Instruments, Japan) after staining and drying.

\section{Metabolomics and fatty acid contents}

For testae flavonoids-metabolomics, testae lipidmetabolomics, and embryo untarget-metabolomics, freeze-dried coats were extracted with differentially buffer according to the instructions of METWARE (Wuhan, China) and BGI (Wuhan, China). Sample extracts were analyzed using an LC-ESI-MS/MS system (HPLC, Shimpack UFLC SHIMADZU CBM30A system; MS, Applied Biosystems 6500 Q TRAP; MS, API 6500 Q TRAP), and quantified as previously described [67]. The metabolite contents were scaled using Z-score of peak area (mean value of three biological replications) in the heatmap.

Extraction and analysis of seed fatty acids was performed as described previously [68].

\section{RNA sequencing, data processing, and gene annotation}

Seed coats of WT and wsc were harvested at DAF20, DAF40, and DAF60 in 2017 according to their developmental stage and color; these samples were then subject to RNA-seq using an Illumina HiSeq platform at the BGI (Wuhan, China). A $3 \mu$ g sample of total RNA from each sample was used to enrich messenger RNA and construct complementary DNA libraries. Thus, use of the internal software SOAPnuke to filter reads yielded a total of $6.66 \mathrm{~Gb}$ of clean bases on average for each repeat. HISAT was used for the mapping step on the basis of a peanut synthetic tetraploid reference genome containing A. duranensis and A. ipaensis [69](https://peautbase.org/). The sequence data have been uploaded in the SRA database of National Center for Biotechnology Information under the accession number of PRJNA497474. A total of 61,903 expressed genes were detected including a total of 7336 predicted as new. We merged a series of novel coding transcripts with reference ones to generate a complete reference set subsequent to the detection of novel transcripts and then mapped clean reads onto this using the software Bowtie2 [70], RSEM was used to calculate gene expression levels for each sample [71]. DEGseq was applied to identify differentially expression genes between samples via algorithms, and resultant $P$ values were adjusted using a Benjamini and Hochberg's correction to control for FDR. Genes determined via
DESeq as having an adjusted $\mathrm{P}$ value $<0.05$ were defined as DEGs. Gene annotation was performed using the software Blast2GO. Functional interpretation of these DEGs was further completed by assigning them to metabolic pathways using KEGG annotation. The WGCNA R package was used to do the coexpression anlysis as described in previous publications [72, 73]. The gene expression was scaled using Z-score of FPKM (mean value of three biological replications) in the heatmap.

\section{qRT-PCR analysis}

Reverse transcriptions and qRT-PCR were carried out as described before according to Wan et al. [23].

\section{Statistical analysis}

The statistical significance was calculated by Student's $t$ test. The gene expression values of FPKM were scaled to Z-score to draw the heatmap of transcriptome and metabolome. Each experiment was repeated three or six times.

\section{Supplementary information}

Supplementary information accompanies this paper at https://doi.org/10. 1186/s12870-020-02383-7.

Additional file 1. Flavonoids detected in peanut testae.

Additional file 2. Testae lipid metabolome data between WSC and WT.

Additional file 3. Embryo metabolome data between wsC and WT.

Additional file 4. Cotyledon metabolism and fatty acid contents in wsC and WT. (A) Enriched KEGG pathways of cotyledon metabolism in WSC and WT. (B) Fatty acid contents in WSC and WT.

Additional file 5. DEGs identified between wsC and WT. (XLS 8402 kb) Additional file 6. Gene ontoloty enrichment of the DEGs between wsC and WT.

Additional file 7. DEGs KEGG pathways between wsC and WT.

Additional file 8. Gene-specific primers used in qRT-PCR analysis.

Additional file 9. GRT-PCR validation of DEGs between wSC and WT. (A) Transcript levels of 17 genes with average FPKM value $\geq 2.5$. The $y$-axis showed relative gene expression levels analyzed by qRT-PCR and RNASeq. WT qRT-PCR (rose columns) and wsC qRT-PCR (white columns) corresponding to qRT-PCR expression data. WT RNA-Seq (rose lines) and wsC RNA-Seq (gray lines) refer to RNA-seq data. The data presented here are mean values from three repetitions. Error bars represent standard error (SE) $(n=3)$. (B) Comparison of gene expression ratios from qRT-PCR and RNA-Seq data. RNA-Seq log2 values of the expression ratio (y-axis) are plotted against the three different developmental stages ( $x$-axis). The gene expression was scaled using Z-score of FPKM (mean value of three biological replications) in the heatmap.

Additional file 10. Comparison of glycolysis and citrate cycles between WSC and WT. (A) Comparison of glycolysis between WSC and WT; (1) hexokinase; (2) glucosephosphate isomerase; (3) phosphofructokinase; (4) aldolase; (5) triose phosphofructokinase; (6) glyceraldehyde phosphate dehydrogenase; (7) phosphoglycerate kinase; (8) phosphoglyceromutase; (9) enolase; (10) pyruvate kinase; (11) non-enzymatic reaction; (12) lactate dehydrogenase; (13) pyruvate decarboxylase; (14) alcohol dehydrogenase. (B) Comparison of the citrate cycle between wsC and WT; (pre), pyruvate dehydrogenase complex; (1) citrate synthase; (2) aconitase; (3) aconitase; (4) isocitrate dehydrogenase; (5) a-ketoglutarate dehydrogenase; (6) succinyl CoA synthase; (7) succinate dehydrogenase; (8) funarase; (9) 
malate dehydrogenase. Gene expression was scaled using Z-scores of FPKM for mean valued of three biological replicates in heatmaps.

Additional file 11. FPKM values for DEGs in glycolysis and the TCA cycle. (A) Expression of hexokinase genes. (B) Expression of pyruvate kinases genes. (C) Expression of phosphofructokinase genes. (D) Expression of pyruvate dehydrogenase component genes. (E) Expression of aconitate hydratase genes. (F) Expression of citrate synthase genes. (G) Expression of isocitrate dehydrogenase genes. $(H)$ Expression of succinate dehydrogenase genes. (I) Expression of malate dehydrogenase genes.

Additional file 12. Expression profiles of sucrose synthesis and transport genes in wsC and WT. SS, sucrose synthase; SUT, sucrose transport protein; STP; sugar transporter protein; ERD, early response to dehydration transporters; SWEET, Sugars Will Eventually be Exported Transporters.

Additional file 13. The expression of phytohormone-synthesis pathway genes in wsC and WT. (A) Heatmap and FPKM values of different expressed BR synthesis pathway genes between wsC and WT. (B) Heatmap and FPKM values of the differently expressed GA synthesis pathway genes between ws and WT. (C) Heatmap and FPKM values of differently expressed JA synthesis pathway genes between wsC and WT. BR6OX1, brassinosteroid-6-oxidase 1; BAS1, PHYB-4 ACTIVATION-TAGGED SUPPRESSOR 1; KAO, ent-kaurenoic acid hydroxylase; GA20X, gibberellin 2-oxidase; GA30x, gibberellin 3-beta-dioxygenase; PLA1, phospholipase A1; AOC, allene oxide cyclase; OPR, 12-oxophytodienoic acid reductase; MFP2: multifunctional protein 2. The gene expression was scaled using Zscore of FPKM (mean value of three biological replications) in the heatmap.

Additional file 14. The expression of mutipl-phytohormones signaling pathway genes in wsc and WT. (A) Heatmap and FPKM values for differently expressed ABA signaling pathway genes between wsC and WT. (B) Heatmap and FPKM values of differently expressed auxin synthesis pathway genes between wsC and WT. (C) Heatmap and FPKM values of differently expressed BR signaling pathway genes between wsC and WT. (D) Heatmap and FPKM values of differently expressed CTK signaling pathway genes between wsC and WT. (E) Heatmap and FPKM values of different expressed GA signaling pathway genes between wsc and WT. (F) Heatmap and FPKM values of differently expressed JA signaling pathway genes between wsc and WT. (G) Heatmap and FPKM values of differently expressed SA signaling pathway genes between wsC and WT. The gene expression was scaled using Z-score of FPKM (mean value of three biological replications) in the heatmap.

Additional file 15. Differentially expressed MYB, bHLH, and WD40 factors between wsc and WT. (A) Heatmap and expression levels of differentially expressed MYB transcription factors between wsC and WT. (B) Heatmap and expression levels of differentially expressed bHLH factors between wsC and WT. (C) Heatmap and expression levels of differentially expressed WD40 factors between WsC and WT. The gene expression was scaled using Z-score of FPKM (mean value of three biological replications) in the heatmap.

Additional file 16. Expression and annotation of common DEGs at the three developmental stages assessed in this study.

Additional file 17. The growing observation of wsC and WT.

Additional file 18. Expression and annotation of stitching genes between seed specified and common DEGs at the three developmental stages assessed in this study.

Additional file 19. Sequence alignment of Araip.MHR6K between wsC and WT.

Additional file 20. WSC losses affect multiple primary and secondly metabolism pathways. Mutant of WSC leads to a coordinated increase in transcript and metabolic levels of carbon metabolism pathways and hormone synthesis and signaling pathways while the flavonoids metabolism pathway was redirected. Up- and down-regulated genes and metabolites are shown in red and green, respectively.

\section{Abbreviations}

ABA: Abscisic acid; ANR: Anthocyanidin reductase; AUX: Auxin: BR: Brassinosteroid; $\mathrm{CHI}$ : Chalcone isomerase; CHS: Chalcone synthase;
CTK: Cytokinin; DAF: Days after flowering; DEG: Differentially expressed gene; DFR: Dihydroflavonol 4-reductase; EBGs: Early biosynthetic genes; F3H: Flavanone 3-hydroxylase; F3'H: Flavonoid 3'-hydroxylase; F3'5'H: Flavonoid 3',5'-hydroxylase; FDR: False discovery rate; FLS: Flavonol synthase; FPKM: Fragments per Kilobase Million; GA: Gibberellin; HISAT: Hierarchical Indexing for Spliced Alignment of Transcripts; JA: Jasmonic acid; KEGG: Kyoto Encyclopedia of Genes and Genomes; LAR: Leucoanthocyanidin reductase; LDOX: Leucoanthocyanidin dioxygenase; LBGs: Late biosynthetic genes; MBW: MYB-bHLH-WD40; MeJA: Methyl jasmonate; MTs: Methyltransferases; PA: Proanthocyanidin; POD: Peroxidase; PPOD: Polyphenol oxidase; qRT-PCR: Quantitative reverse-transcription PCR: SA: Salicylic acid; SUTs: $\mathrm{H}^{+} /$sucrose symporters; TBO: Toluidine blue O; TCA: Tricarboxylic acid; TSTs: The $\mathrm{H}^{+} /$sucrose antiporters located on tonoplast; UFGT: UDP-glucose:flavonoid 3-O-glucosyl transferase; wsc: White seed coat peanut mutant; WT: Wild Type

\section{Acknowledgements}

We thank Dr. Juncheng Zhang (Huazhong Agricultural University, China) for critically revising the English in this paper, as well as Dr. Jianbo Jian (BGI) for assistance with RNA-seq analyses.

\section{Authors' contributions}

LYW carried out the laboratory work in collaboration with YL1 and YL2, LYY, $X W$, MKP. JHF and RKV performed the data analysis and interpretation. LYW prepared the figures and tables. LYW and BSL drafted the manuscript. All authors read and approved the final manuscript.

\section{Funding}

This research was funded by grants from the National Natural Science Foundation of China (Nos. 31971820 and 31301256), the Innovation Program of the Chinese Academy of Agricultural Sciences, the Central Public-Interest Scientific Institution Basal Research Fund, and the China Agriculture Research System (No. CARS-13), the National Key Research and Development Program of China (No. 2018YFD1000900) and the Jiangxi Agriculture Research System (No. JCARS-18). Funders did not participate in the design of the study, analysis of the results and writing of the manuscript, but provided financial support for the manuscript.

\section{Availability of data and materials}

Transcriptome sequencing data are available in the SRA database of National Center for Biotechnology Information under the accession number of PRJNA497474 (https://www.ncbi.nlm.nih.gov/bioproject/PRJNA497474).

\section{Ethics approval and consent to participate}

Not applicable.

\section{Consent for publication}

Not applicable.

\section{Competing interests}

The authors have no conflict of interests to declare.

\section{Author details}

${ }^{1}$ Key Laboratory of Biology and Genetic Improvement of Oil Crops, Ministry of Agriculture, Oil Crops Research Institute of Chinese Academy of Agricultural Sciences, Wuhan, China. ${ }^{2}$ Key Laboratory of Crop Physiology, Ecology and Genetic Breeding, Ministry of Education, Jiangxi Agricultural University, Nanchang, China. ${ }^{3}$ Southern Regional Collaborative Innovation Center for Grain and Oil Crops in China, Nanchang, China. ${ }^{4}$ College of Agronomy, Jiangxi Agricultural University, Nanchang, China. ${ }^{5}$ Center of Excellence in Genomics, International Crops Research Institute for the Semi-Arid Tropics, Hyderabad, India. ${ }^{6}$ School of Plant Biology and Institute of Agriculture, The University of Western, Australia, Crawley, WA, Australia.

Received: 13 February 2020 Accepted: 2 April 2020

Published online: 15 April 2020

\section{References}

1. Yao LH, Jiang YM, Shi J, Tomás-Barberán FA, Datta N, Singanusong R, Chen SS. Flavonoids in food and their health benefits. Plant Foods Hum Nutr. 2004;59:113-22. 
2. Owens D, Alerding A, Crosby K, Bandara A, Westwood J, Winkel B. Functional analysis of a predicted flavonol synthase gene family in Arabidopsis. Plant Physiol. 2008;147:1046-61.

3. Veitch N, Grayer R. Flavonoids and their glycosides, including anthocyanins. Nat Prod Rep. 2011;28:1626-95.

4. Byrne P, Darrah L, Snook M, Wiseman B, Widstrom N, Moellenbeck D, Barry B. Maize silk-browning, maysin content, and antibiosis to the corn earworm, Helicoverpa zea (Boddie). Maydica. 1996;41:13-8.

5. Choi J, Islam M, Ali M, Kim E, Kim Y, Jung H. Effects of C-glycosylation on anti-diabetic, anti-Alzheimer's disease and anti-inflammatory potential of apigenin. Food Chem Toxicol. 2014;64:27-33.

6. Czemmel S, Stracke R, Weisshaar B, Cordon N, Harris N, Walker A, Robinson SP, Bogs J. The grapevine R2R3-MYB transcription factor VVMYBF1 regulates flavonol synthesis in developing grape berries. Plant Physiol. 2009;151:1513-30.

7. Kuhn B, Geisler M, Bigler L, Ringli C. Flavonols accumulate asymmetrically and affect auxin transport in Arabidopsis. Plant Physiol. 2011;156:585-95.

8. Stracke R, Jahns O, Keck M, Tohge T, Niehaus K, Fernie A, Weisshaar B. Analysis of PRODUCTION OF FLAVONOL GLYCOSIDES-dependent flavonol glycoside accumulation in Arabidopsis thaliana plants reveals MYB11-, MYB12- and MYB111-independent flavonol glycoside accumulation. New Phytol. 2010;188:985-1000.

9. Mondal S, Roy S. Genome-wide sequential, evolutionary, organizational and expression analyses of phenylpropanoid biosynthesis associated MYB domain transcription factors in Arabidopsis. J Biomol Struct Dyn. 2018;36: $1577-601$.

10. Li S. Transcriptional control of flavonoid biosynthesis: fine-tuning of the MYB-bHLH-WD40 (MBW) complex. Plant Signal Behav. 2014:9:e27522.

11. Franceschi $V$, Grimes $H$. Induction of soybean vegetative storage proteins and anthocyanins by low-level atmospheric methyl jasmonate. Proc Natl Acad Sci U S A. 1991:88:6745-9.

12. Feys B, Benedetti C, Penfold C, Turner J. Arabidopsis mutants selected for resistance to the phytotoxin coronatine are male sterile, insensitive to methyl jasmonate, and resistant to a bacterial pathogen. Plant Cell. 1994;6:751-9.

13. Wang $Y$, Liu W, Jiang $H$, Mao Z, Wang $N$, Jiang $S$, Xu H, Yang G, Zhang Z, Chen X. The R2R3-MYB transcription factor MdMYB24-like is involved in methyl jasmonate-induced anthocyanin biosynthesis in apple. Plant Physiol Biochem. 2019;139:273-282.

14. Devoto A, Ellis C, Magusin A, Chang H, Chilcott C, Zhu T, Turner JG. Expression profiling reveals COl1 to be a key regulator of genes involved in wound- and methyl jasmonate-induced secondary metabolism, defence, and hormone interactions. Plant Mol Biol. 2005;58:497-513.

15. Loreti E, Povero G, Novi G, Solfanelli C, Alpi A, Perata P. Gibberellins, jasmonate and abscisic acid modulate the sucrose-induced expression of anthocyanin biosynthetic genes in Arabidopsis. New Phytol. 2008;179:1004-16.

16. Shan X, Zhang Y, Peng W, Wang Z, Xie D. Molecular mechanism for jasmonate-induction of anthocyanin accumulation in Arabidopsis. J Exp Bot. 2009:60:3849-60

17. Peng Z, Han C, Yuan L, Zhang K, Huang H, Ren C. Brassinosteroid enhances jasmonate-induced anthocyanin accumulation in Arabidopsis seedlings. J Integr Plant Biol. 2011;53:632-40.

18. Gabetta B, Fuzzati N, Griffini A, Lolla E, Pace R, Ruffilli T, Peterlongo F. Characterization of proanthocyanidins from grape seeds. Fitoterapia. 2000;71:162-75.

19. Dixon R, Xie D, Sharma S. Proanthocyanidins - a final frontier in flavonoid research? New Phytol. 2005;165:9-28.

20. Santos-Buelga C, Scalbert A. Proanthocyanidins and tannin-like compounds - nature, occurrence, dietary intake and effects on nutrition and health. J Sci Food Agric. 2000;80:1094-117.

21. Chukwumah $Y$, Walker $L$, Verghese M. Peanut skin color: a biomarker for total polyphenolic content and antioxidative capacities of peanut cultivars. Int J Mol Sci. 2009;10:4941-52.

22. Wan L, Li B, Pandey M, Wu Y, Lei Y, Yan L, Dai $X$, Jiang $H$, Zhang J, Wei G, Varshney RK, Liao B. Transcriptome analysis of a new peanut seed coat mutant for the physiological regulatory mechanism involved in seed coat cracking and pigmentation. Front Plant Sci. 2016;7:1491.

23. Wan L, Li B, Lei Y, Yan L, Ren X, Chen Y, Dai X, Jiang H, Zhang J, Guo W, Chen A, Liao B. Mutant Transcriptome Sequencing Provides Insights into Pod Development in Peanut (Arachis hypogaea L.). Front Plant Sci. 2017:8:1900.

24. Vishwanath S, Delude C, Domergue F, Rowland O. Suberin: biosynthesis, regulation, and polymer assembly of a protective extracellular barrier. Plant Cell Rep. 2015;34:573-86.
25. Li-Beisson Y, Shorrosh B, Beisson F, Andersson M, Arondel V, Bates P, Baud S, Bird D, Debono A, Durrett TP, Franke RB, Graham IA, Katayama K, Kelly AA, Larson T, Markham JE, Miquel M, Molina I, Nishida I, Rowland O, Samuels L, Schmid KM, Wada H, Welti R, Xu C, Zallot R, Ohlrogge J. Acyl-lipid metabolism. Arabidopsis Book. 2013;11:e0161.

26. Kuhn N, Guan L, Dai Z, Wu B, Lauvergeat V, Gomesm E, Li SH, Godoy F, Arce-Johnson P, Delrot $S$. Berry ripening: recently heard through the grapevine. J Exp Bot. 2014;65:4543-59.

27. Ai T, Naing A, Arun M, Lim S, Kim C. Sucrose-induced anthocyanin accumulation in vegetative tissue of Petunia plants requires anthocyanin regulatory transcription factors. Plant Sci. 2016;252:144-50.

28. Bihmidine S, Julius B, Dweikat I, Braun D. Tonoplast sugar transporters (SbTSTs) putatively control sucrose accumulation in sweet sorghum stems. Plant Signal Behav. 2016;11:e1117721.

29. Milne R, Byrt C, Patrick J, Grof C. Are sucrose transporter expression profiles linked with patterns of biomass partitioning in sorghum phenotypes? Front Plant Sci. 2013:4:223.

30. Bihmidine S, Bake R, Hoffner C, Braun D. Sucrose accumulation in sweet sorghum stems occurs by apoplasmic phloem unloading and does not involve differential sucrose transporter expression. BMC Plant Biol. 2015;15:186.

31. Leach K, Tran T, Slewinski T, Meeley R, Braun D. Sucrose transporter2 contributes to maize growth, development, and crop yield. J Integr Plant Biol. 2017;59:390-408.

32. Eom J, Chen L, Sosso D, Julius B, Lin I, Qu X, Braun DM, Frommer WB. SWEETs, transporters for intracellular and intercellular sugar translocation. Curr Opin Plant Biol. 2015;25:53-62

33. Tao Y, Cheung L, Li S, Eom J, Chen L, Xu Y, Perry K, Frommer WB, Feng L. Structure of a eukaryotic SWEET transporter in a homotrimeric complex. Nature. 2015:527:259-63.

34. Mizuno H, Kasuga S, Kawahigashi H. The sorghum SWEET gene family: stem sucrose accumulation as revealed through transcriptome profiling. Biotechnol Biofuels. 2016;9:127

35. Rai A, Umashankar S, Rai M, Kiat L, Bing J, Swarup S. Coordinate regulation of metabolite glycosylation and stress hormone biosynthesis by TT8 in Arabidopsis. Plant Physiol. 2016:171:2499-515

36. Baudry A, Caboche M, Lepiniec L. TT8 controls its own expression in a feedback regulation involving TTG1 and homologous MYB and bHLH factors, allowing a strong and cell-specific accumulation of flavonoids in Arabidopsis thaliana. Plant J. 2006:46:768-79.

37. Gonzalez A, Mendenhall J, Huo Y, Lloyd A. TTG1 complex MYBs, MYB5 and $T 2$, control outer seed coat differentiation. Dev Biol. 2009;325: $412-21$.

38. Clevenger J, Chu Y, Scheffler B, Ozias-Akins P. A developmental transcriptome map for allotetraploid Arachis hypogaea. Front Plant Sci. 2016;7:1446.

39. Hemm M, Rider S, Ogas J, Murry D, Chapple C. Light induces phenylpropanoid metabolism in Arabidopsis roots. Plant J. 2004;38:765-78.

40. Masclaux-Daubresse C, Clement G, Anne P, Routaboul J, Guiboileau A, Soulay F, Shirasu K, Yoshimoto K. Stitching together the multiple dimensions of autophagy using metabolomics and transcriptomics reveals impacts on metabolism, development, and plant responses to the environment in Arabidopsis. Plant Cell. 2014;26:1857-77.

41. Wei S, Li X, Gruber M, Feyissa B, Amyot L, Hannoufa A. COP9 signalosome subunit 5A affects phenylpropanoid metabolism, trichome formation and transcription of key genes of a regulatory tri-protein complex in Arabidopsis. BMC Plant Biol. 2018;18:134.

42. Spelt C, Quattrocchio F, Mol J, Koes R. ANTHOCYANIN1 of petunia controls pigment synthesis, vacuolar $\mathrm{pH}$, and seed coat development by genetically distinct mechanisms. Plant Cell. 2002;14:2121-35.

43. Carey C, Strahle J, Selinger D, Chandler V. Mutations in the pale aleurone color1 regulatory gene of the Zea mays anthocyanin pathway have distinct phenotypes relative to the functionally similar TRANSPARENT TESTA GLABRA1 gene in Arabidopsis thaliana. Plant Cell. 2004:16:450-64.

44. Gonzalez A, Zhao M, Leavitt J, Lloyd A. Regulation of the anthocyanin biosynthetic pathway by the $\Pi \mathrm{TG} 1 / \mathrm{bHLH} / \mathrm{Myb}$ transcriptional complex in Arabidopsis seedlings. Plant J. 2008;53:814-27.

45. Zhao M, Morohashi K, Hatlestad G, Grotewold E, Lloyd A. The TTG1bHLH-MYB complex controls trichome cell fate and patterning through direct targeting of regulatory loci. Development. 2008;135: 1991-9. 
46. Lloyd A, Brockman A, Aguirre L, Campbell A, Bean A, Cantero A, Gonzalez A. Advances in the MYB-bHLH-WD repeat (MBW) pigment regulatory model: addition of a WRKY factor and co-option of an anthocyanin MYB for betalain regulation. Plant Cell Physiol. 2017;58:1431-41.

47. Winkel-Shirley B. Flavonoid biosynthesis. A colorful model for genetics, biochemistry, cell biology, and biotechnology. Plant Physiol. 2001;126:485-93.

48. Xiao J, Capanoglu E, Jassbi A, Miron A. Advance on the flavonoid Cglycosides and health benefits. Crit Rev Food Sci Nutr. 2016;56(Suppl 1): S29-45

49. Wasternack C, Hause B. Jasmonates: biosynthesis, perception, signal transduction and action in plant stress response, growth and development. An update to the 2007 review in annals of botany. Ann Bot. 2013;111:1021-58.

50. Wang S, Chen C, Wang C, Chen P. Resveratrol content in strawberry fruit is affected by preharvest conditions. J Agric Food Chem. 2007;55:8269-74.

51. Wang K, Jin P, Cao S, Shang H, Yang Z, Zheng Y. Methyl jasmonate reduces decay and enhances antioxidant capacity in Chinese bayberries. J Agric Food Chem. 2009;57:5809-15.

52. Flores G, Blanch G, Del Castillo M. Effect of postharvest methyl jasmonate treatment on fatty acid composition and phenolic acid content in olive fruits during storage. J Sci Food Agric. 2017;97:2767-72.

53. Wasternack C, Song S. Jasmonates: biosynthesis, metabolism, and signaling by proteins activating and repressing transcription. J Exp Bot. 2017;68:1303-21.

54. Dombrecht B, Xue G, Sprague S, Kirkegaard J, Ross J, Reid J, Fitt GP, Sewelam N, Schenk PM, Manners JM, Kazan K. MYC2 differentially modulates diverse jasmonate-dependent functions in Arabidopsis. Plant Cell. 2007; 19:2225-45.

55. Fernandez-Calvo P, Chini A, Fernandez-Barbero G, Chico J, Gimenez-Ibanez S, Geerinck J, Eeckhout D, Schweizer F, Godoy M, Franco-Zorrilla JM, Pauwels L, Witters E, Puga MI, Paz-Ares J, Goossens A, Reymond P, De Jaeger G, Solano R. The Arabidopsis bHLH transcription factors MYC3 and MYC4 are targets of JAZ repressors and act additively with MYC2 in the activation of jasmonate responses. Plant Cell. 2011;23:701-15.

56. Montiel G, Zarei A, Korbes A, Memelink J. The jasmonate-responsive element from the ORCA3 promoter from Catharanthus roseus is active in Arabidopsis and is controlled by the transcription factor AtMYC2. Plant Cell Physiol. 2011;52:578-87.

57. Hong G, Xue X, Mao Y, Wang L, Chen X. Arabidopsis MYC2 interacts with DELLA proteins in regulating sesquiterpene synthase gene expression. Plant Cell. 2012;24:2635-48.

58. Zhai Q, Yan L, Tan D, Chen R, Sun J, Gao L, Dong MQ, Wang Y, Li C. Phosphorylation-coupled proteolysis of the transcription factor MYC2 is important for jasmonate-signaled plant immunity. PLoS Genet. 2013;9:e1003422

59. Song S, Qi T, Wasternack C, Xie D. Jasmonate signaling and crosstalk with gibberellin and ethylene. Curr Opin Plant Biol. 2014;21:112-9.

60. Asami T, Nakano T, Fujioka S. Plant brassinosteroid hormones. Vitam Horm. 2005;72:479-504

61. Noguchi T, Fujioka S, Choe S, Takatsuto S, Yoshida S, Yuan H, Feldmann KA, Tax FE. Brassinosteroid-insensitive dwarf mutants of Arabidopsis accumulate brassinosteroids. Plant Physiol. 1999;121(3):743-52.

62. Choe S, Schmitz RJ, Fujioka S, Takatsuto S, Lee MO, Yoshida S, Feldmann KA, Tax FE. Arabidopsis brassinosteroid-insensitive dwarf12 mutants are semidominant and defective in a glycogen synthase kinase 3 beta-like kinase. Plant Physiol. 2002;130(3):1506-15.

63. Mora-García S, Vert G, Yin Y, Caño-Delgado A, Cheong H, Chory J. Nuclear protein phosphatases with Kelch-repeat domains modulate the response to brassinosteroids in Arabidopsis. Genes Dev. 2004;18(4):448-60.

64. Sun Y, Fan XY, Cao DM, Tang W, He K, Zhu JY, He JX, Bai MY, Zhu S, Oh E, Patil S, Kim TW, Ji H, Wong WH, Rhee SY, Wang ZY. Integration of brassinosteroid signal transduction with the transcription network for plant growth regulation in Arabidopsis. Dev Cell. 2010;19(5):765-77.

65. Figueiredo D, Batista R, Roszak P, Hennig L, Kohler C. Auxin production in the endosperm drives seed coat development in Arabidopsis. Elife. 2016;5: e20542.

66. Qu C, Fu F, Lu K, Zhang K, Wang R, Xu X, Wang M, Lu J, Wan H, Zhanglin T, $\mathrm{Li}$ J. Differential accumulation of phenolic compounds and expression of related genes in black- and yellow-seeded Brassica napus. J Exp Bot. 2013; 64(10):2885-98.

67. Dong T, Han R, Yu J, Zhu M, Zhang Y, Gong Y, Li Z. Anthocyanins accumulation and molecular analysis of correlated genes by metabolome and transcriptome in green and purple asparaguses (Asparagus officinalis, L.). Food Chem. 2019;271:18-28.
68. Poirier Y, Ventre G, Caldelari D. Increased flow of fatty acids toward betaoxidation in developing seeds of Arabidopsis deficient in diacylglycerol acyltransferase activity or synthesizing medium-chain-length fatty acids. Plant Physiol. 1999;121:1359-66.

69. Bertioli DJ, Cannon SB, Froenicke L, Huang G, Farmer AD, Cannon EK, Liu X, Gao D, Clevenger J, Dash S, Ren L, Moretzsohn MC, Shirasawa K, Huang W, Vidigal B, Abernathy B, Chu Y, Niederhuth CE, Umale P, Araújo AC, Kozik A, Kim KD, Burow MD, Varshney RK, Wang X, Zhang X, Barkley N, Guimarães PM, Isobe S, Guo B, Liao B, Stalker HT, Schmitz RJ, Scheffler BE, Leal-Bertioli SC, Xun X, Jackson SA, Michelmore R, Ozias-Akins P. The genome sequences of Arachis duranensis and Arachis ipaensis, the diploid ancestors of cultivated peanut. Nat Genet. 2016:48:438-46.

70. Langmead B, Salzberg S. Fast gapped-read alignment with bowtie 2. Nat Methods. 2012;9:357-9.

71. Li B, Dewey C. RSEM: accurate transcript quantification from RNA-Seq data with or without a reference genome. BMC Bioinform. 2011;12:323.

72. Langfelder P, Horvath S. WGCNA: an R package for weighted correlation network analysis. BMC Bioinform. 2008;9:559.

73. Langfelder $\mathrm{P}$, Horvath S. Fast $\mathrm{R}$ functions for robust correlations and hierarchical clustering. J Stat Softw. 2012;46:1-17.

\section{Publisher's Note}

Springer Nature remains neutral with regard to jurisdictional claims in published maps and institutional affiliations.

\section{Ready to submit your research? Choose BMC and benefit from:}

- fast, convenient online submission

- thorough peer review by experienced researchers in your field

- rapid publication on acceptance

- support for research data, including large and complex data types

- gold Open Access which fosters wider collaboration and increased citations

- maximum visibility for your research: over $100 \mathrm{M}$ website views per year

At BMC, research is always in progress.

Learn more biomedcentral.com/submissions 